\title{
Análise de fatores críticos de sucesso de projetos: um estudo de caso no setor varejista
}

\author{
Sandra Morioka ${ }^{\mathrm{a} *}$, Marly Monteiro de Carvalho ${ }^{\mathrm{b}}$ \\ a*sandra.morioka@usp.br, EPUSP, Brasil \\ ${ }^{\mathrm{b}}$ marlymc@usp.br, EPUSP, Brasil
}

\begin{abstract}
Resumo
0 objetivo deste trabalho é compreender a relação entre os fatores críticos de sucesso em gestão de projetos e o resultado dos projetos, a partir de uma perspectiva contingencial, contemplando os diferentes tipos de projetos. A pesquisa de campo desenvolvida em uma empresa do setor de varejo foi feita em duas fases: análise documental e entrevistas para diagnóstico da situação de gerenciamento de projetos na empresa e pesquisa do tipo survey com questionário fechado aplicado aos principais envolvidos. Foram obtidos 43 questionários respondidos. Um dos resultados da pesquisa indica o impacto significativo da tipologia de projetos sobre o desempenho de determinados fatores críticos de sucesso, evidenciando a importância de se definir critérios objetivos de classificação de projetos. Foi possível ainda visualizar graficamente a relação entre o desempenho de cada dimensão de sucesso e os benefícios percebidos, a partir da adaptação do diagrama de Kano de satisfação de clientes usada anteriormente na síntese da literatura.
\end{abstract}

Palavras-chave

Sucesso de projeto. Fatores críticos de sucesso de projetos. Tipologia de projeto. Diagrama de Kano.

\section{Introdução}

0 sucesso, para uma empresa orientada a projetos, está diretamente ligado aos resultados obtidos em cada um de seus projetos, já que esses constituem o negócio fundamental e as competências essenciais da empresa (KERZNER, 2006). Por outro lado, para empresas cujo foco está em outros processos, a execução de projetos tem como principal função sustentar as atividades estratégicas e não rotineiras da organização como, por exemplo, em uma empresa do ramo varejista.

É possível fazer uma distinção entre sucesso de gestão de projeto e sucesso de projeto (DE WIT, 1988). 0 sucesso em gestão de projetos está principalmente associado ao sucesso da atuação direta do gerente de projetos, aplicando as ferramentas dessa disciplina. Essa discussão está basicamente associada à tríade restrição de qualquer projeto, composta por escopo, prazo e custo. Nesse sentido, um projeto é considerado bem-sucedido se o seu escopo proposto inicialmente foi cumprido, se foi entregue dentro do prazo estipulado e se os custos decorrentes da sua execução não excederam o orçamento previsto para esse projeto. Dado que existe um conflito intrínseco entre cada uma das facetas da tríade, o desafio do gerente de projeto consiste em administrar esse trade off de forma a maximizar os benefícios decorrentes da realização do projeto. Assim, o projeto que teve um gerenciamento de sucesso apresenta uma alta eficiência na aplicação dos recursos disponíveis durante a execução do projeto.

Em contraposição ao sucesso de gerenciamento de projeto, o sucesso de projetos refere-se aos objetivos e benefícios previstos pelo projeto para a organização como um todo. Nesse sentido, trata-se da eficácia de uma determinada iniciativa atrelada ao cumprimento de seu objetivo inicial, possibilitando que a empresa usufrua dos benefícios previstos pelo projeto. 
É válido ressaltar que essas duas facetas de sucesso nem sempre estão correlacionadas. Embora seja de se esperar que o sucesso em gestão de projetos leve ao sucesso do próprio projeto para a empresa, nem sempre isso ocorre. Por outro lado, também há que se considerar que os fatores críticos podem ser influenciados pelo tipo de projeto (SHENHAR; DVIR, 2007).

Nesse contexto, o objetivo deste trabalho é compreender a relação entre os fatores críticos de sucesso em gestão de projetos e seu impacto no resultado dos projetos, a partir de uma perspectiva contingencial, contemplando os diferentes tipos de projetos. A pesquisa foi desenvolvida em uma empresa de varejo de grande porte no período de 2009 a 2010. Este artigo está estruturado em cinco seções. $\mathrm{Na}$ síntese do quadro teórico, apresentada na seção 2 , são explorados os principais aspectos relacionados a fatores críticos de sucesso em projetos. A seção 3 apresenta o detalhamento da abordagem metodológica utilizada na pesquisa. Os resultados da pesquisa são apresentados na seção 4 e, em seguida (seção 5), o artigo traz as principais conclusões.

\section{Fatores críticos de sucesso e avaliação de sucesso de projetos}

Os pilares dessa pesquisa são os fatores críticos de sucesso, a partir de uma perspectiva contingencial, e os critérios utilizados para a avaliação de sucesso nos projetos.

\subsection{Fatores críticos de sucesso}

De forma geral, fatores críticos de sucesso podem ser definidos como o número limitado de áreas que devem apresentar resultados minimamente satisfatórios para garantir o sucesso do desempenho competitivo organizacional. Por isso, essas áreas devem estar sob constante atenção da gerência, garantindo o resultado final bem-sucedido (ROCKART, 1979).

$\mathrm{Na}$ área de gestão de projetos, os trabalhos pioneiros sobre a temática de fatores críticos de sucesso são os dos pesquisadores Murphy, Baker e Fisher (MURPHY; BAKER; FISHER, 1974; BAKER; MURPHY; FISHER, 1988) e dos pesquisadores Pinto e Slevin (SLEVIN; PINTO, 1986; PINTO; SLEVIN, 1987; 1988a b; 1989).

Murphy, Baker e Fisher (1974) fizeram um levantamento junto a gerentes de projeto e levantaram dez fatores fortemente relacionados ao sucesso/fracasso de projetos. Posteriormente, identificaram 23 características de gerenciamento de projetos necessárias mas não suficientes para o sucesso (BAKER; MURPHY; FISHER, 1988) .

Pinto e Slevin conduziram vários trabalhos sobre sucesso e fracasso em projetos na década de 1980 (SLEVIN: PINTO, 1986: PINTO; SLEVIN, 1987; 1988a, b; 1989). Os autores basearam parte de suas conclusões em um levantamento realizado junto aos membros do PMl em que se atribuia relevância a dez fatores críticos de sucesso: missão do projeto, apoio da alta administração, plano/cronograma do projeto, consulta ao cliente, pessoal, atividades e tecnologia em apoio ao projeto, aceitação do cliente, monitoramento e realimentação, canais de comunicação e resolução de problemas. Eles também elaboraram um modelo para definição do critério de sucesso em projeto que considera as métricas clássicas de sucesso (triângulo de ferro), mas introduziu outras dimensões, como eficácia, satisfação e uso. Pinto e Slevin (1988b) também colocaram a perspectiva do ciclo de vida na temática dos fatores críticos de sucesso em projetos, pois identificaram que os fatores variam de acordo com a fase do ciclo de vida do projeto, exceto a missão do projeto que se mostrou importante em todas as fases do ciclo de vida.

A literatura apresenta diversas formas de tratar esse assunto e cada autor propõe sua série de fatores críticos de sucesso de projetos, sendo que muitos desses fatores se sobrepõem e outros apresentam enfoques distintos (FORTUNE; WHITE, 2006; KERZNER, 2006; COOKE-DAVIES, 2002; LOPES, 2009; entre outros).

Para facilitar o entendimento dos diversos fatores críticos de sucesso apresentados pela literatura, o presente trabalho propõe uma classificação em cinco dimensões de fatores críticos de sucesso: planejamento e controle, natureza do projeto, recursos humanos, stakeholders e meio externo ao projeto (ver Quadro 1).

No entanto, esses fatores críticos de sucesso não devem ser vistos como universais. Os autores israelenses Shenhar e Dvir têm sustentado uma abordagem contingencial (SHENHAR; DVIR, 1996; SHENHAR, 2001; SHENHAR et al., 2002). Shenhar (2001) sugere que o mito do one-size-fits-all (os fatores são universais) conduz, frequentemente, para o fracasso e propõe que é necessário observar as contingências impostas pelos diferentes tipos e características dos projetos (one-size-does-not-fit-all). Dessa forma, os diferentes tipos de projeto devem ser considerados na discussão sobre fatores críticos de sucesso de projetos e avaliação de sucesso de projetos. Há diversos critérios a serem adotados para uma classificação de projetos adequada (SHENHAR et al., 2002; EVARISTO, FENEMA; 1999, BLOMQUIST, 2004 apud PATAH; CARVALHO; 2009; SABBAG 1999 apud CARVALHO; RABECHINI JUNIOR, 2008; SHENHAR, DVIR, 2007 apud RABECHINI JUNIOR, CARVALHO, 
Quadro 1. Dimensões de fatores críticos de sucesso de projeto.

\begin{tabular}{|c|c|c|c|}
\hline \multicolumn{4}{|c|}{ Fatores criticos de sucesso de projeto } \\
\hline Grupo & Dimensão & Observações & Exemplos \\
\hline \multirow{2}{*}{ 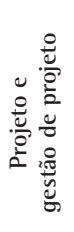 } & $\begin{array}{l}\text { Planejamento e } \\
\text { controle }\end{array}$ & $\begin{array}{l}\text { - Atuação direta do gerente de } \\
\text { projeto }\end{array}$ & $\begin{array}{l}\text { Objetivos claros e realistas; Gestão de mudanças efetiva; } \\
\text { Gestão de riscos efetiva; Controle e monitoramento efetivos; } \\
\text { Organização clara e simples para o projeto; Controle gerencial } \\
\text { dinâmico e eficiente }\end{array}$ \\
\hline & Natureza do projeto & $\begin{array}{l}\text { - Referência às características do } \\
\text { projeto, evidenciando a necessidade } \\
\text { de uma tipologia adequada }\end{array}$ & $\begin{array}{l}\text { Tecnologia conhecida; Valor percebido do projeto; Projeto } \\
\text { de grande porte, alto nível de complexidade, muitas pessoas } \\
\text { envolvidas, longa duração }\end{array}$ \\
\hline \multirow{2}{*}{ 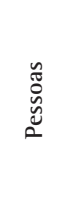 } & Recursos humanos & $\begin{array}{l}\text { - Preocupação com a equipe do } \\
\text { projeto }\end{array}$ & $\begin{array}{l}\text { Boa comunicação e bom feedback; Equipe de projeto suficiente } \\
\text { e qualificada; Gerente de projetos bem preparado; Boa liderança; } \\
\text { Treinamentos adequados; Motivação e seleção da equipe }\end{array}$ \\
\hline & Stakeholders & $\begin{array}{l}\text { - Verificação dos envolvidos no } \\
\text { projeto }\end{array}$ & $\begin{array}{l}\text { Suporte da alta diretoria; Envolvimento de clientes e usuários; } \\
\text { Bom desempenho de fornecedores, contratados e consultores; } \\
\text { Diversos pontos de vista }\end{array}$ \\
\hline 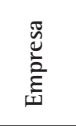 & Meio externo & $\begin{array}{l}\text { - Características externas ao âmbito } \\
\text { do projeto em si }\end{array}$ & $\begin{array}{l}\text { Adaptação, cultura e estrutura da organização; Estabilidade } \\
\text { política; Ferramentas e métodos de gestão de projetos bem } \\
\text { escolhidos; Compreensão do ambiente do projeto (contexto); } \\
\text { Sistema de informações gerenciais confiável }\end{array}$ \\
\hline
\end{tabular}

Fonte: adaptado de Fortune e White (2006) e Lopes (2009).

Quadro 2. Classificação de projetos.

\begin{tabular}{|c|c|}
\hline Literatura & Critérios de classificação de projetos \\
\hline Shenhar et al. (2002) & $\begin{array}{l}\text { - Complexidade do escopo do sistema: assembly, system e array } \\
\text { - Incerteza tecnológica: baixa, média, alta e superalta }\end{array}$ \\
\hline Evaristo e Fenema (1999) & $\begin{array}{l}\text { - Projetos isolados ou conjunto de projetos } \\
\text { - Número de locais em que o(s) projeto(s) está(ão) sendo desenvolvido(s) }\end{array}$ \\
\hline $\begin{array}{l}\text { Blomquist (2004) apud Patah e Carvalho } \\
\text { (2009) }\end{array}$ & - Grau de inovação: derivativo, de plataforma, breakthrough e P\&D \\
\hline $\begin{array}{c}\text { Sabbag (1999) apud Carvalho e Rabechini } \\
\text { Junior (2008) }\end{array}$ & $\begin{array}{l}\text { - Complexidade } \\
\text { - Singularidade } \\
\text { - Rigor das metas }\end{array}$ \\
\hline $\begin{array}{l}\text { Shenhar e Divir (2007) apud Rabechini } \\
\text { Junior e Carvalho (2009) }\end{array}$ & $\begin{array}{l}\text { "Diamante NCTP" } \\
\text { - Novidade: derivativo, plataforma, inédito } \\
\text { - Complexidade: conjunto, sistema, grupo } \\
\text { - Tecnologia: baixa, média, alta, superalta } \\
\text { - Passo: regular, rápido/competitivo, tempo-crítico e urgente }\end{array}$ \\
\hline McFarlan (1984) & $\begin{array}{l}\text { "Cranfiel Grid" } \\
\text { - Impacto para o presente: baixo e alto } \\
\text { - Impacto para o futuro: baixo e alto }\end{array}$ \\
\hline Carvalho e Rabechini Junior (2010) & $\begin{array}{l}\text { "Modelo 14" - Projetos classificados em: } \\
\text { - Imediato } \\
\text { - Impacto } \\
\text { - Inovação } \\
\text { - Integração }\end{array}$ \\
\hline
\end{tabular}

Fonte: elaboração própria.

2009; McFARLAN, 1984; CARVALHO; RABECHINI JUNIOR, 2010). Exemplos modelos de tipologia de projetos são apresentados no quadro 2. Conforme pode ser observado no Quadro 2, os diversos modelos apresentados na literatura não se contrapõem, mas se complementam entre si.

\subsection{Avaliação de sucesso de projetos}

Conforme mencionado anteriormente, as métricas clássicas utilizadas para a avaliação de sucesso em projetos inclui a tríplice restrição, ou triângulo de ferro: escopo, prazo e custo, mas, com a evolução da literatura, outras dimensões de sucesso foram incorporadas.

Shenhar e Dvir (2007) subdividem os possíveis critérios de avaliação de sucesso em cinco dimensões: eficiência, impacto para o cliente, impacto para a equipe, negócio e sucesso imediato e, por fim, preparação para o futuro (ver Quadro 3).

Conforme explicitado no Quadro 3, há autores que fazem a distinção entre os dois aspectos de sucesso 
de projeto: o do projeto e o da gestão do projeto (por exemplo: DE WIT, 1988). Enquanto o sucesso no gerenciamento do projeto está focado principalmente na conformidade de escopo, prazo e cronograma acordados entre os stakeholders, o sucesso do projeto em si avalia também na prática os benefícios para a empresa, equipe e clientes do projeto.

Para complementar a discussão sobre as cinco dimensões de sucesso, pode-se fazer uso de uma adaptação do modelo de Kano de satisfação do cliente. 0 diagrama de Kano estuda o grau de satisfação do cliente em função do desempenho de um determinado critério, classificando-o em: itens básicos de qualidade, itens de desempenho e itens de encantamento ao consumidor (KANO et al., 1984; CARVALHO, 2001; HÖLZING, 2008). Neste trabalho, propõe-se uma modificação do diagrama de Kano para o sucesso em projetos: ao invés de se considerar o grau de satisfação do cliente no eixo y, adota-se a intensidade do benefício aproveitado pela empresa em face do desempenho nas dimensões de sucesso nesta seção (ver Figura 1).

Analogamente ao comportamento dos itens de encantamento de um determinado produto, a dimensão preparação para o futuro possui um potencial significativo para trazer benefícios relevantes à empresa. Por outro lado, uma eventual falha nesse sentido não implica necessariamente em prejuízo para a organização no momento presente.

Já as dimensões de impacto para o negócio e sucesso imediato, bem como a dimensão impacto para o cliente possuem um comportamento análogo aos fatores de desempenho do modelo de Kano. Isso porque quanto maior a satisfação dos usuários em

Quadro 3. Avaliação de sucesso de projeto.

\begin{tabular}{|c|c|c|c|}
\hline & \multicolumn{3}{|r|}{ Avaliação de sucesso de projeto } \\
\hline & & Dimensão & Observações \\
\hline \begin{tabular}{|c|}
$\begin{array}{c}\text { Efeito de } \\
\text { curto prazo }\end{array}$ \\
\end{tabular} & 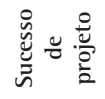 & Eficiência & Considera a tríade restrição clássica (escopo, prazo e custo) \\
\hline & \multirow{4}{*}{ 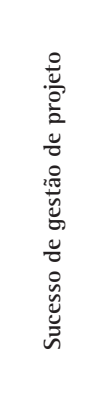 } & $\begin{array}{l}\text { Impacto para o } \\
\text { cliente }\end{array}$ & $\begin{array}{l}\text { Considera critérios como satisfação das necessidades, melhoria da qualidade de vida } \\
\text { (SHENHAR; DVIR, 2007), medidas técnicas e funcionalidades (SHENHAR; DVIR, 2007; } \\
\text { VERZUH, 2000) e suavidade na transferência da equipe para o cliente final (BRYDE, 2003 }\end{array}$ \\
\hline & & $\begin{array}{l}\text { Impacto para } \\
\text { equipe }\end{array}$ & $\begin{array}{l}\text { Verifica como o projeto afeta os membros da equipe, marcando o evento na vida profissional } \\
\text { das pessoas de forma positiva ou negativa, dependendo do desenvolvimento e resultado do } \\
\text { projeto (SHENHAR; DVIR, 2007). }\end{array}$ \\
\hline & & $\begin{array}{l}\text { Negócio e } \\
\text { impactos } \\
\text { imediatos }\end{array}$ & $\begin{array}{l}\text { Analisa as consequências diretas causadas pelo desenvolvimento do projeto, verificando } \\
\text { fatores como aumento de volume de vendas, de receita e de lucratividade (SHENHAR; DVIR, } \\
\text { 2007), bem como R01, competitividade e desempenho de mercado (MUNNS; BJEIMI, 1996) } \\
\text { e capacidade do projeto em contribuir para a melhoria contínua da empresa (BRYDE, 2003) }\end{array}$ \\
\hline $\begin{array}{c}\text { Efeito de } \\
\text { longo prazo }\end{array}$ & & $\begin{array}{c}\text { Preparação para } \\
\text { o futuro }\end{array}$ & $\begin{array}{l}\text { Analisa os efeitos de longo prazo possibilitados pelo projeto, fazendo referência à } \\
\text { preparação de infraestrutura da empresa para o futuro e criação de novas oportunidades } \\
\text { (SHENHAR; DVIR, 2007). }\end{array}$ \\
\hline
\end{tabular}

Fonte: baseado em Shenhar e Dvir (2007).

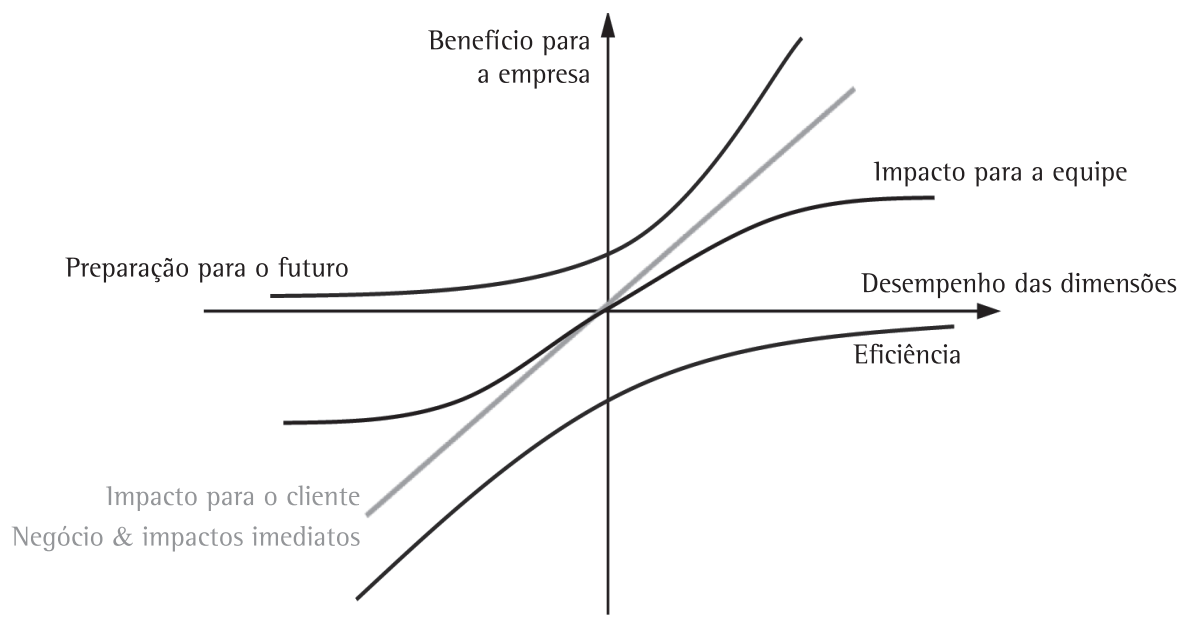

Figura 1. Modelo de Kano adaptado para as dimensões de sucesso de projeto. Fonte: adaptada de Kano et al. (1984) 
relação às entregas dos projetos e quanto melhor os impactos imediatos para a empresa, maiores serão os benefícios percebidos pela organização como um todo. Ao mesmo tempo, porém, projetos que tiveram insucesso nessas dimensões têm seus resultados negativos também percebidos pela empresa, que deixa de obter o retorno previsto inicialmente pelo projeto.

Assim como os fatores básicos previstos pelo modelo de Kano, a dimensão de sucesso referente à eficiência do projeto tem potencial apenas de causar insatisfação, caso ela não seja atendida. Dessa forma, prazo, custo e escopo devem estar de acordo com os parâmetros definidos previamente com os patrocinadores do projeto para que não sejam desperdiçados recursos humanos e financeiros.

Já a dimensão "satisfação da equipe" apresenta um comportamento particular, distinto dos três descritos por Kano em seu diagrama. Propõe-se que existe um ponto de saturação quanto aos benefícios percebidos pela empresa. Projetos encerrados sem grandes conflitos e ruídos entre os envolvidos, trazendo os resultados esperados, proporcionam valor à empresa, já que os membros da equipe do projeto estarão motivados e preparados para executar suas próximas tarefas de projeto com mais eficiência e eficácia. Porém, a relação positiva entre impacto para a equipe e benefício para a empresa tem um ponto de saturação, já que o quanto as pessoas poderão agregar no seu próximo projeto é limitado, dado que cada projeto é um caso particular e possui desafios únicos a serem enfrentados. 0 mesmo vale para uma eventual insatisfação da equipe diante da execução do projeto. A frustração dos conflitos e insucessos de um projeto anterior pode prejudicar, mesmo que limitadamente, o desempenho do projeto seguinte.

\subsection{Síntese do quadro teórico}

0 quadro síntese dessa revisão da literatura é apresentado da Figura 2. Nela observa-se o intuito de analisar o impacto dos fatores críticos de sucesso nos resultados, a partir de uma perspectiva contingencial.

\section{Metodologia}

Neste trabalho optou-se por estruturar a pesquisa em quatro fases. A primeira fase foi a pesquisa bibliográfica, contemplando tipologia de projetos, fatores críticos de sucesso em gerenciamento de projetos e avaliação de sucesso em projetos. A pesquisa de campo foi desenvolvida em uma empresa do setor de varejo, em duas fases: (a) diagnóstico da situação de gerenciamento de projetos na empresa, a partir da análise documental e entrevistas, e (b) uma pesquisa do tipo survey com questionário fechado aplicado aos principais envolvidos na atividade de projetos na empresa. A última fase foi a análise e discussão dos resultados obtidos, conforme ilustra a Figura 3.

Para a realização de uma análise dos fatores críticos de sucesso foi conduzido, inicialmente, um levantamento teórico baseado em três pilares, conforme discutido na seção anterior.

Em seguida foi selecionada uma empresa do setor de varejo para o desenvolvimento da pesquisa de campo, já que a organização se encontrava em um momento em que as diretrizes de gestão de projetos estavam em processo de amadurecimento por meio da consolidação do PMO implantado na empresa. $\mathrm{Na}$ primeira etapa foi realizada uma análise crítica do portfólio de projetos da empresa. Para tanto, foram tomados como base indicadores extraídos do banco de dados da empresa, dentre os quais podem ser citados orçamento, duração e maturidade de escopo. Além disso, o trabalho traz uma discussão sobre a tipologia de projetos adotada pela empresa, confrontando-a com a literatura pesquisada sobre o assunto e com sua realidade em termos de gerenciamento de projetos.

Além da consulta ao banco de dados da empresa, o trabalho conta com dados empíricos obtidos por

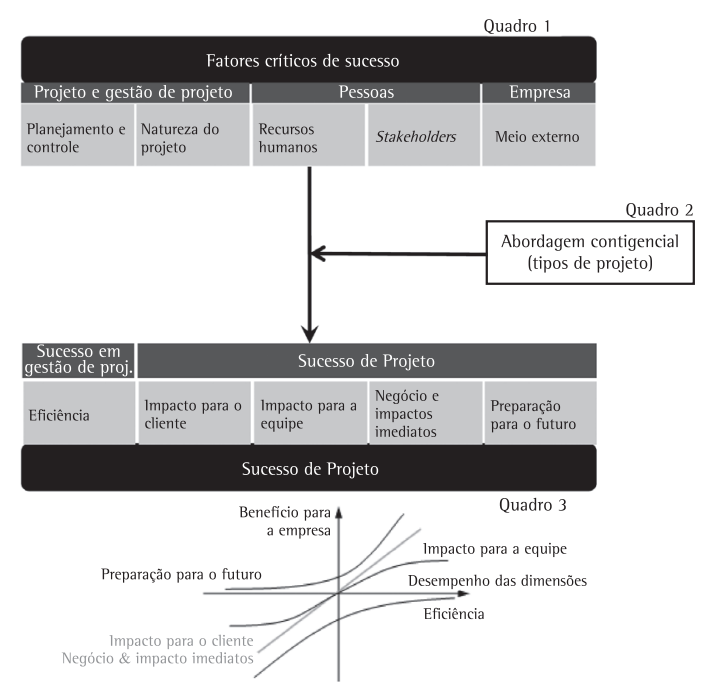

Figura 2. Síntese do quadro teórico.

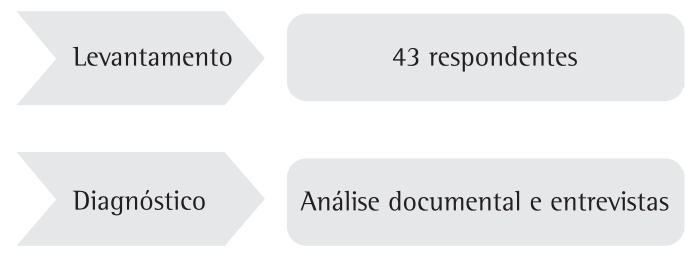

Figura 3. Projeto da pesquisa de campo. 
meio de um questionário sobre avaliação de sucesso e fatores críticos de sucesso de projetos enviado aos principais envolvidos com projetos na empresa. Para o preenchimento do questionário foi pedido que o respondente escolhesse o último projeto concluído no qual ele teve envolvimento significativo. A preferência por projetos concluídos é justificada pelo fato de que, dessa forma, as respostas dadas poderão trazer uma visão holística do projeto, desde o planejamento, passando pela execução e chegando até sua finalização. Já a exigência de que fosse escolhido o último projeto concluído se faz necessária uma vez que sem essa restrição as pessoas tenderiam a escolher os projetos que foram mais bem-sucedidos, resultando em um viés nos resultados. Assim, baseado em um determinado projeto, o questionário teve como objetivo identificar, dentre alguns fatores críticos de sucesso de projetos apontados pela literatura, a intensidade com que cada um deles está de fato sendo praticado pela empresa. A partir desse resultado é possível identificar as principais lacunas de desempenho desses fatores críticos de sucesso de projeto abordados no questionário. Além disso, o questionário busca também investigar como as pessoas avaliam o sucesso do projeto selecionado.

0 questionário está estruturado em três grandes blocos, sendo que para cada pergunta o respondente assinala uma escala tipo Likert de 1 a 5 . Dessa forma, foram avaliados:

(i) visão geral do projeto: valor que esse projeto representa em comparação aos demais projetos da empresa, risco atrelado à sua execução e avaliação geral do sucesso do projeto;

(ii) fatores críticos de sucesso de projeto: definição clara e controle de escopo/cronograma/ orçamento, motivação e comprometimento da equipe do projeto, comunicação clara e eficaz entre os envolvidos, envolvimento de clientes/usuários durante o desenvolvimento do projeto, envolvimento do PMO, bom desempenho de fornecedores e terceiros, apoio da alta diretoria, disponibilidade de ferramentas para o controle de projetos e, por fim, utilização de uma metodologia corporativa de gestão de projetos;

(iii) avaliação de sucesso de projeto (desempenho e peso): cumprimento da linha base do projeto, satisfação gerada ao usuário, contribuição para a vida profissional dos membros da equipe do projeto, bem como os ganhos imediatos e os impactos de longo prazo gerados pelo projeto.

0 questionário foi enviado aos seis membros do PMO, bem como a dez representantes de cada uma das cinco diretorias, totalizando 56 questionários enviados. Com uma parte dos questionários enviada via correio eletrônico e outra entregue impressa, a taxa de retorno, com 43 questionários preenchidos, foi satisfatória.

\section{Apresentação e discussão dos resultados}

A pesquisa foi desenvolvida em uma empresa de varejo de grande porte, presente em diversos estados brasileiros, que comercializa produtos para casa, vestuário e produtos financeiros, como cartão de crédito, garantias e empréstimos. Nesse cenário, o departamento de gestão de projetos, exercendo o papel de um project management office (PMO), é responsável pela manutenção do portfólio de projetos da empresa, consolidando e apresentando à diretoria executiva as principais informações relacionadas aos projetos de melhoria e inovação de processos.

A análise dos tipos de projeto foi realizada com base na análise documental dos projetos do portfólio da empresa. Já a análise dos fatores críticos de projeto e dos critérios de sucesso foi feita sobre um levantamento realizado com base na percepção dos stakeholders envolvidos, conforme apresentado a seguir.

\subsection{Fatores críticos de sucesso e sucesso: resultado do levantamento}

0 questionário foi distribuido a 56 respondentes, dez para cada uma das quatro diretorias, dez para superintendência e seis para os analistas do PMO. Dessa forma pretendeu-se atingir as principais pessoas envolvidas em projetos e inseridas nas diversas áreas de negócio da empresa. Foi solicitado que o questionário fosse respondido com base no último projeto finalizado no qual o respondente teve envolvimento considerável. 0 resultado obtido foi baseado em 43 questionários respondidos, o que representa uma taxa de retorno de $76,8 \%$ do total de questionários enviados às diversas áreas.

Como pode ser observado na Figura 4, o fator crítico de sucesso apresentado no questionário que obteve maior pontuação, em média, foi o de motivação e comprometimento do gerente e da equipe de projeto durante o desenvolvimento do projeto. Já o segundo fator crítico de sucesso mais desempenhado pelos projetos da empresa, conforme o resultado do questionário aplicado, está relacionado ao envolvimento de clientes ou usuários das entregas do projeto durante suas diversas fases do ciclo de vida. Como, em geral, os clientes dos projetos pertencem à empresa, essa proximidade ao usuário se dá de forma natural, já que o conhecimento técnico das áreas de negócio usuárias dos produtos do projeto é também insumo necessário para a definição do escopo do projeto. Dessa forma, as próprias pessoas que são clientes do projeto pertencem também de certa forma à equipe do projeto. 
Fatores críticos de sucesso

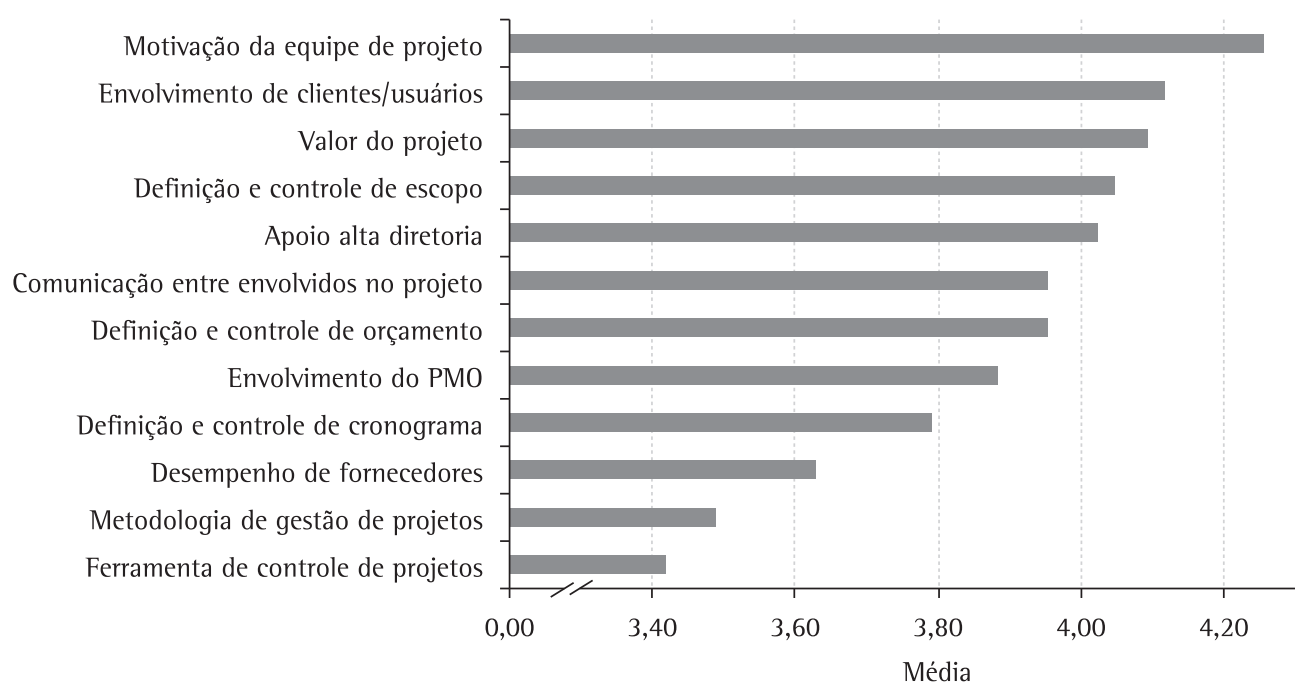

Figura 4. Média da avaliação de desempenho dos fatores críticos de sucesso de projeto. Escala de Likert de 1 a 5.

Por outro lado, dentre os fatores críticos de sucesso de projetos listados no questionário, o que apresentou um desempenho mais tímido durante o desenvolvimento do projeto foi a utilização da metodologia de gestão de projetos e das ferramentas de controle de projetos. Como o PMO está em fase de consolidação, a metodologia e a ferramenta de gestão de projetos definidas ainda não estão completamente incorporadas aos processos da empresa.

Um importante ponto de atenção na Figura 5 é que, ao observar a dimensão de sucesso da eficiência no desenvolvimento do projeto, fica evidente que, apesar de ter sido considerada um dos mais importantes critérios na avaliação final do projeto, seu desempenho ainda tem muito a melhorar. Além disso, a dimensão referente ao impacto para o cliente foi considerada fundamental na avaliação final do projeto, bem como tem uma posição de destaque também quanto ao seu desempenho. Isso conflui com o resultado do desempenho dos fatores críticos de sucesso de projetos, já que este indicou um intenso envolvimento de clientes e usuários finais ainda durante o desenvolvimento do projeto, garantindo sua satisfação ante as entregas do projeto.

\subsection{Abordagem contingencial: classificação dos tipos de projeto com base na análise documental}

Os projetos analisados nesse trabalho são iniciativas provenientes dos diversos departamentos da empresa, atendendo a demandas da superintendência, bem como das quatro diretorias executivas: comercial, financeira, de operações e de controladoria. Nesse contexto, os projetos tratados estão principalmente ligados à revisão e melhoria de processos, mas também podem buscar a introdução de novos produtos e serviços ou a adaptação a mudanças na legislação vigente. Vale também ressaltar que projetos relacionados à infraestrutura de $\mathrm{Tl}$ e projetos de reforma das instalações das lojas não serão contemplados nas análises apresentadas, já essas demandas são muito específicas. Assim, a maioria dos projetos tratados (98\%) possui desenvolvimentos da área de tecnologia de informação (criação/adaptação de softwares), uma vez que qualquer mudança operacional tem impacto nos sistemas utilizados.

A classificação dos projetos utilizada pela organização estudada para os projetos contemplados pelo PMO prevê a divisão de seus projetos em três tipos.

TIP0 1: projetos estratégicos com intenso envolvimento do PMO, que possuem maior evidência perante a diretoria executiva e a empresa como um todo;

TIPO 2: projetos estratégicos com envolvimento moderado do PMO;

TIPO 3: projetos de cunho operacional, cuja responsabilidade no sucesso ou fracasso recai sobre o departamento demandante desse projeto.

Retomando-se o grid estratégico como forma de classificar projetos, esses três tipos de projeto adotados pela empresa podem ser visualizados na Figura 6.

Como evidenciado na Figura 7, os projetos de tipo 1 são aqueles poucos projetos (13\%) que têm importância bastante estratégica para a empresa, justificando sua expressiva participação nos gastos com 


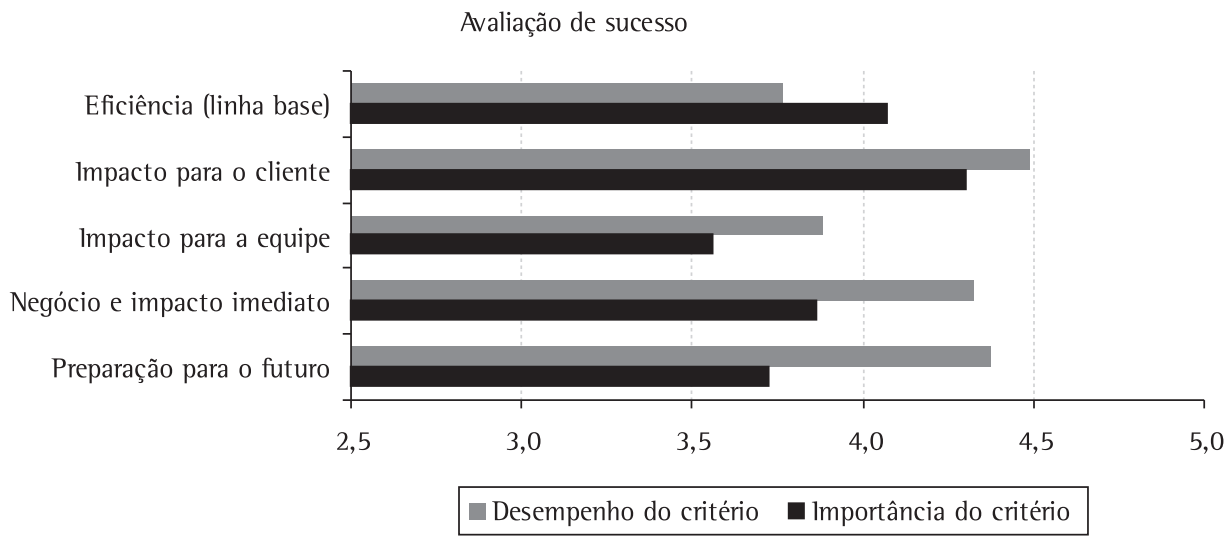

Figura 5. Resultado da avaliação de sucesso dos projetos da empresa.

projetos (36\%). São nesses projetos que a participação do PMO é bastante intensa, já que se trata de projetos que buscam benefícios mais representativos e de maior grau de complexidade.

A Figura 8 apresenta a relação entre a duração, a multidisciplinaridade (número de departamentos envolvidos) e o orçamento dos projetos. Como pode ser observada, essa classificação não revela tendências evidentes quanto aos parâmetros considerados. Isso porque a classificação dos projetos nesses três tipos foi realizada conforme a percepção do grau de complexidade e de importância estratégica de cada projeto, extraída da discussão entre o PMO e as áreas de negócio demandantes dos projetos. Dessa forma, os critérios utilizados para essa categorização de projetos acabam sendo subjetivos.

Seria razoável considerar também outros critérios para a classificação dos projetos, tais como intensidade de inovação tecnológica (BLOMQUIST, 2004; SHENHAR, DVIR, 2007), grau de inovação (SHENHAR; DVIR; 2007), intensidade da urgência de entrega do projeto (SHENHAR; DVIR, 2007), intensidade do risco atrelado à execução do projeto e o impacto sobre o cliente final da empresa. lsso porque esses parâmetros justificariam também a necessidade de uma maior evidência perante a diretoria executiva. Tais parâmetros, porém, não são monitorados para os projetos da empresa.

\subsection{Discussão dos resultados: a abordagem de Kano adaptada}

0 uso do diagrama de Kano permitiu relacionar as dimensões de sucesso em projetos e seus benefícios para a organização, além de trazer uma síntese da perspectiva presente e futura, conforme sugere McFarlan (1984). A interpretação dos dados obtidos

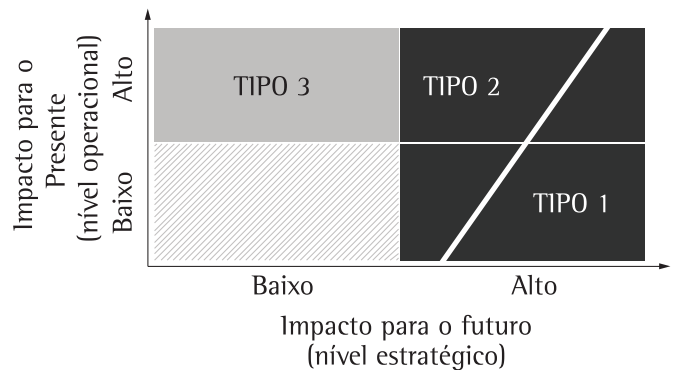

Figura 6. Aplicação do grid estratégico de McFarlan (1984) para os tipos de projeto da empresa

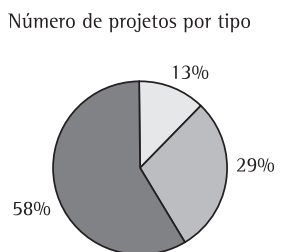

Orçamento por tipo de projeto

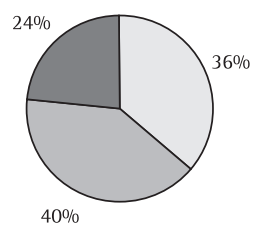

$$
\square \text { Tipo } 1 \quad \square \text { Tipo } 2 \square \text { Tipo } 3
$$

Figura 7. Estatísticas dos projetos por tipo.

segundo o modelo de Kano adaptado para as dimensões de avaliação de sucesso de projeto apresenta uma sintese gráfica interessante.

A Figura 9 apresenta a média atual do desempenho dos critérios de sucesso dos projetos abordados no questionário (cores sólidas). Já os pontos preenchidos com cor branca no centro representam um cenário futuro de cada uma dessas dimensões, baseado na percepção de importância atribuída às dimensões de sucesso pelos respondentes da amostra. Nesse sentido, o maior salto de desempenho esperado refere-se à dimensão da eficiência, já que, apesar de o atendimento à linha base do projeto ter apresentado um desempenho inferior às demais dimensões, essa 


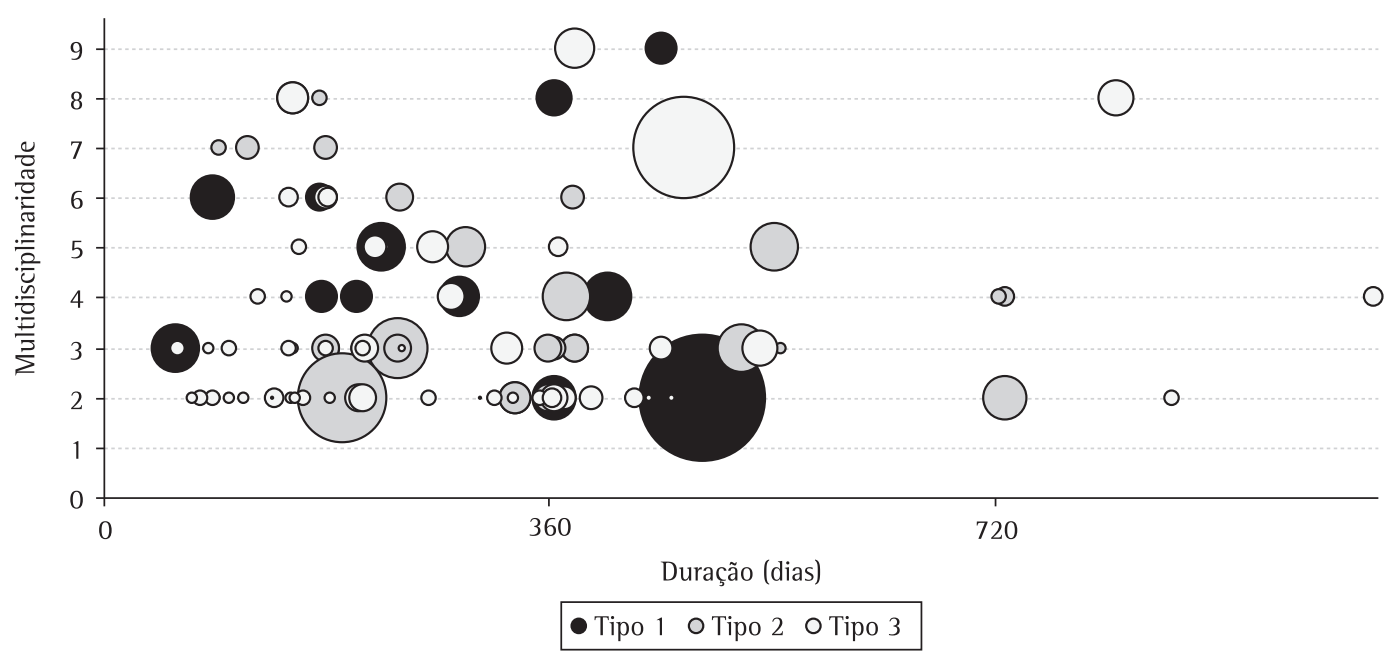

Figura 8. Análise dos tipos de projeto adotados pela empresa. Nota: o tamanho da bolha representa o orçamento de cada projeto.

dimensão foi considerada uma das mais importantes pelos respondentes. Dessa forma, observa-se na organização estudada que o triângulo de ferro ainda é prioridade na organização, conforme sugerem vários autores (SHENHAR; DVIR, 2007; VERZUH, 2000), mas o desempenho ainda está aquém do planejado pela organização.

Observa-se ainda na Figura 9 a percepção de prioridade no que concerne à satisfação do cliente, dimensão considerada de grande importância pelos respondentes. Além disso, a análise dos fatores críticos de sucesso traz também o resultado de que a equipe do projeto não é prioridade para a empresa, já que tanto seu desempenho atual quanto o previsto para o futuro apresentam-se inferiores aos demais. Verifica-se também que a organização investe tempo e esforço para garantir o envolvimento do cliente/usuário dos produtos do projeto durante as fases de desenvolvimento do projeto. Nessa dimensão, há uma convergência entre a atenção dada durante a condução do projeto (a equipe procura de fato envolver clientes e usuários), a importância atribuída ao critério (essa dimensão foi considerada a de maior importância na avaliação de sucesso de projeto) e o desempenho real nesse critério (a dimensão que obteve melhor desempenho foi a de satisfação do usuário).

Para aprofundar a análise dos dados obtidos pelo questionário, foi utilizada a ferramenta estatística MINITAB 15 para realizar estudos referentes às possiveis correlações entre os fatores críticos de sucesso e a avaliação de sucesso do projeto. Como dados de entrada, foram consideradas não somente as respostas ao questionário mas também informações

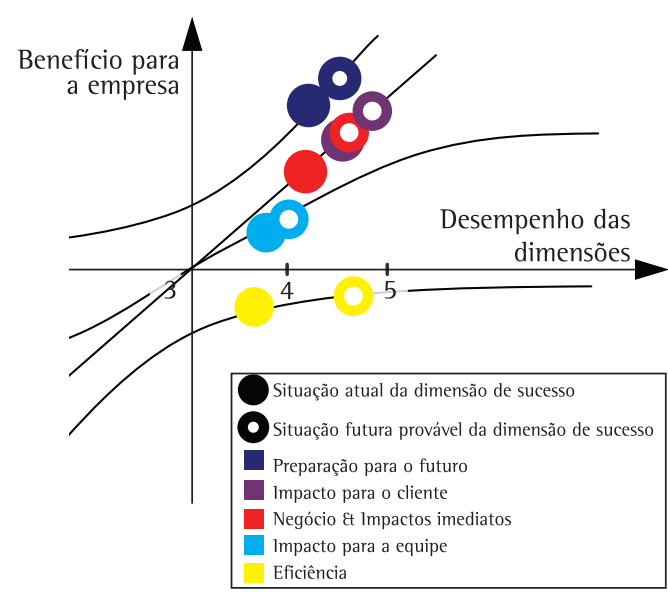

Figura 9. Aplicação do modelo de Kano para o desempenho das dimensões de sucesso de projetos.

sobre os projetos escolhidos no questionário, coletados do banco de dados da empresa. Esses dados extras são referentes ao tipo de projeto $(1,2$ ou 3$)$ e à multidisciplinaridade (número de departamentos envolvidos), bem como à duração, ao número de entregas e ao orçamento do projeto, considerando também os respectivos desempenhos em relação à linha base.

Explicando 73,0\% da variabilidade dos dados, a análise da correlação entre os fatores críticos de sucesso abordados pelo questionário e algumas informações sobre os projetos disponíveis no banco de dados da empresa apresentou uma forte correlação dos dados em três grupos (ver Figura 10). Observa-se, portanto, que a classificação dos projetos impacta não só 


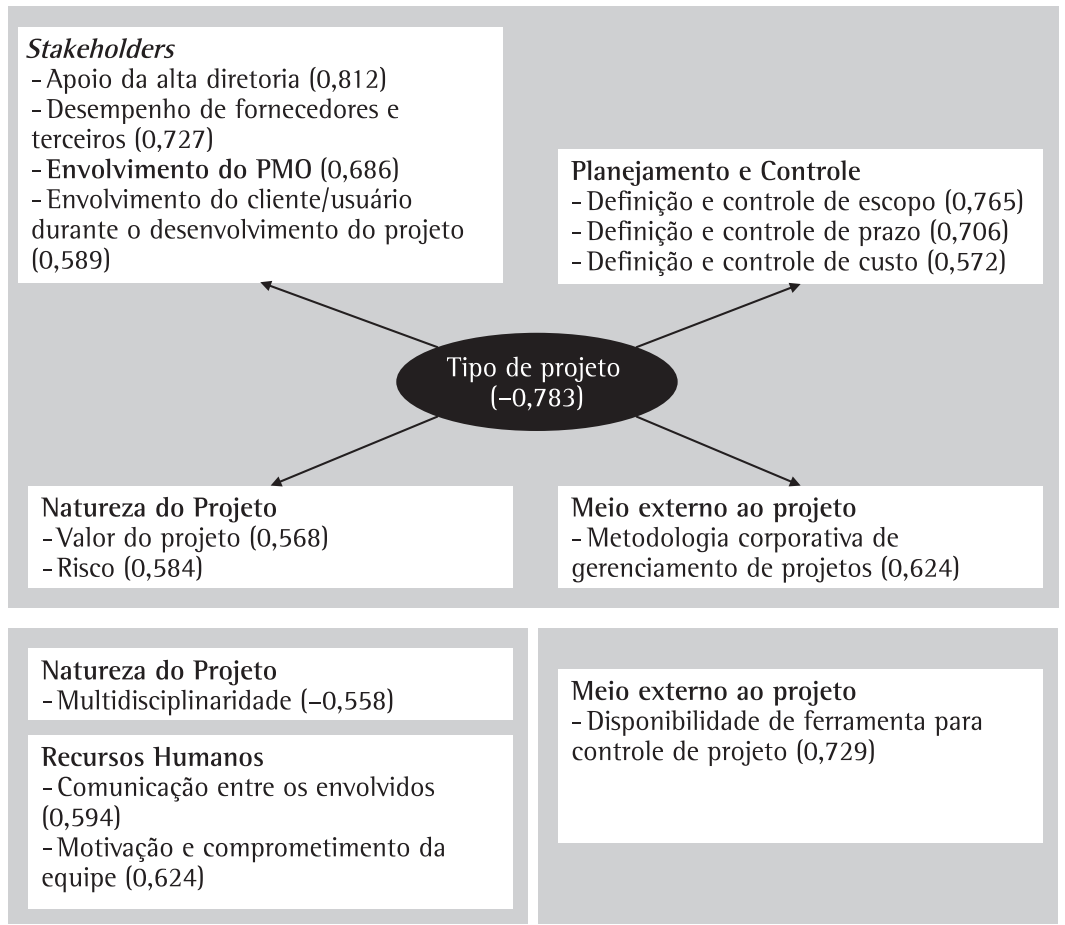

Figura 10. Correlação entre os fatores críticos de sucesso abordados no questionário, agrupados por dimensão de FCS de projeto.

nos resultados do projeto, como sugere a literatura (SHENHAR, 2001; SHENHAR; DVIR, 2007), mas também na presença dos fatores críticos de sucesso na gestão de projetos. Isso pode ser explicado, na organização estudada, pelo fato de a classificação dos tipos de projeto determinar, por definição, o grau de envolvimento do PMO no desenvolvimento do projeto. Dessa forma, a presença de fatores críticos de sucesso é de fato afetada no projeto, como, por exemplo, o envolvimento da alta diretoria e a utilização da metodologia corporativa de gerenciamento de projeto.

A análise mostrou também que tanto motivação quanto comunicação estão relacionadas entre si, mas negativamente correlacionados à multidisciplinaridade do projeto. Isso pode ser um indicador de que as pessoas estão mais motivadas e se comunicam melhor na execução de projetos que envolvem poucas áreas de negócio. 0 envolvimento de poucos departamentos significa, em geral, que os membros da equipe devem pertencer a departamentos parceiros, com os quais a interação no cotidiano é mais intensa.

Além disso, a análise fatorial teve como resultado que a disponibilidade e consequente utilização de uma ferramenta corporativa formal de controle de projeto são independentes do controle de escopo, cronograma e orçamento do projeto efetivamente realizado. Isso evidencia o fato de que o controle de projeto realizado até então é conduzido de forma mais informal e descentralizada, o que acaba deixando a utilização da ferramenta em segundo plano.

A partir desses três principais grupos de FCS de projeto foram conduzidas as demais análises estatísticas, tomando-se um representante de cada um desses grupos.

Ao analisar a relação entre esses fatores críticos de sucesso de projetos e os resultados do projeto tomados a partir do questionário e das variações em relação à linha base do projeto pôde-se verificar que o único fator que não apresentou influência sobre o sucesso do projeto foi a utilização da ferramenta de controle de projeto. Os demais fatores tiveram influência principalmente sobre as entregas concluídas em relação às previstas, bem como sobre atendimento à linha base, satisfação do usuário, impacto sobre a equipe e os ganhos de longo prazo. Os índices apresentados foram: tipo de projeto $(-0,659)$, motivação e comprometimento dos envolvidos $(0,640)$, percepção de sucesso $(0,561)$ índice de desempenho de entregas $(0,580)$, atendimento à linha base $(0,588)$, impacto para o cliente $(0,728)$, impacto para a equipe $(0,657)$ e ganhos de longo prazo $(0,798)$. Essa análise fatorial teve $71,7 \%$ da variação dos dados explicada. É interessante notar que a percepção do sucesso do projeto está de fato associada ao desempenho da maioria das dimensões de sucesso de projeto abordadas pelo questionário, sendo que apenas o desempenho do 
impacto imediato ao negócio propiciado pelo projeto não apresentou correlação significativa à percepção de sucesso do projeto. Assim, pôde-se concluir que a maioria dos fatores críticos de sucesso de projetos propostos pelo questionário apresentaram influência significativa sobre o sucesso dos projetos conduzidos pela empresa, como previsto pela literatura.

A ferramenta estatística foi utilizada também para o estudo de possíveis relações de dependência dos pesos atribuídos às dimensões de avaliação de sucesso com as características do projeto (valor percebido pela empresa, riscos atrelados à execução do projeto, tipo, multidisciplinaridade, além de orçamento, duração e número de entregas da linha base). Justificando $73,0 \%$ da variabilidade dos dados, a análise trouxe a evidência de que os projetos mais estratégicos da empresa, ou seja, os de tipo 1 e 2, além de estarem caracterizados por maior multidisciplinaridade, maior valor percebido do projeto e também maiores riscos de execução, acabam tendo uma menor importância em relação ao atendimento à linha base do projeto na sua avaliação de sucesso (com índice de correlação de 0,666$)$. Assim, para projetos mais importantes estrategicamente, as pessoas tendem a deixar os parâmetros básicos de gerenciamento de projetos (escopo, prazo e custo) em segundo plano, valorizando outras dimensões de sucesso. Isso pode ser uma indicação de que as pessoas entendem a dificuldade de se manter a linha base de projetos mais estratégicos, já que são, na maioria das vezes, mais complexos. Assim, desvios moderados da linha base do projeto podem ser, então, aceitáveis, se forem compensados e justificados pelos demais benefícios do projeto.

\section{Conclusões}

0 presente trabalho apresenta um quadro teórico que relaciona três pilares teóricos da tipologia de projetos, sucesso de projetos e fatores críticos de sucesso de projetos. Esses assuntos foram bastante abordados na literatura, sendo que a contribuição do presente artigo não está na introdução de novos construtos mas na aplicação desses conceitos em uma empresa do ramo do varejo em que os projetos não são o foco no negócio. Assim, mesmo que o tema não tenha mais relevância para a empresa do que demais processos operacionais relacionados aos pontos de venda, foi possível identificar alguns insights interessantes.

0 estudo evidenciou a relação entre fatores críticos pesquisados e o resultado de projetos, que, nesse estudo de caso, com exceção do uso da ferramenta de controle de projeto, tiveram correlação estatística positiva. 0 resultado destaca principalmente a tipologia de projeto como determinante para a maior parte dos
FCS pesquisados. Assim, observou-se que a tipologia de projetos impacta não só nos resultados do projeto mas também na intensidade com que os fatores críticos de gerenciamento são utilizados nos projetos. Isso evidencia a importância de se adotar uma metodologia adequada para classificar os projetos, reduzindo as chances de distorções e influências políticas.

De forma gráfica, o diagrama de Kano permitiu relacionar as dimensões de sucesso em projetos e seus benefícios para a organização, trazendo tanto o desempenho das dimensões no presente quanto a expectativa para o futuro. 0 diagrama indica que o atendimento à tríade de restrição clássica (escopo/prazo/custo) é um fator mínimo qualificador para o sucesso do projeto, evidenciando a importância de se conseguir um bom desempenho nessa dimensão o mais rápido possível. Apesar da importância das demais dimensões de sucesso (impacto para o cliente, impacto para a equipe, negócio e sucesso imediato e preparação para o futuro), pode-se dizer que, na prática, há uma dificuldade de se medir seu desempenho, exigindo um maior esforço das empresas para seu acompanhamento. Assim, próximos estudos poderiam abordar a discussão de indicadores para esse acompanhamento sob uma perspectiva contingencial, levando em consideração os diferentes tipos de projeto propostos em diferentes contextos.

\section{Referências}

BAKER, B. N.; MURPHY, D. C.; FISHER, D. Factors affecting project success. In: CLELAND, D. 1.; KING, W. R. (Eds.). Project Management Handbook. 2nd ed. New York: Van Nostrand Reinhold, 1988. p. 902-909.

BLOMQUIST, T. Program and Portfólio Managers: Analysis of Roles and Responsibilities. In: SLEVIN, D. P.; CLELAND, D. 1.; PINTO, J. K. (Eds.) Innovations Project Management Research. Pennsivania: Newton Square, 2004.

BRYDE, D. J. Modeling Project Management Performance. International Journal of Quality \& Reliability Management, v. 20, n. 2, p. 229-254, 2003. http:// dx.doi.org/10.1108/02656710310456635

CARVALHO, M. M. Qualidade em Projeto. In: AMATO NETO, J. (Org.). Manufatura classe mundial: conceitos, estratégias e aplicações. São Paulo, 2001. p. 131-145.

CARVALHO, M. M.; RABECHINI JUNIOR, R. Construindo competências para gerenciar projetos: Teoria e prática. 2. ed. São Paulo: Atlas, 2008.

CARVALHO, M. M.; RABECHINI JUNIOR, R. Gestão contingencial de projetos: Usando road maps gerenciais para estabelecer vínculo flexível entre metodologias e tipos de projetos. Mundo Project Management, v. 6, n. 32, p. 66-73, 2010.

COOKE-DAVIES, T. The "real" success factors on projects. International Journal of Projetct Management, v. 20, n. 3, p. 185-190, 2002. http://dx.doi.org/10.1016/ S0263-7863(01)00067-9 
DE WIT, A. Measurement of project success. International Journal of Project Management, v.6, n. 3, p. 164-170, 1988. http://dx.doi.org/10.1016/0263-7863(88)90043-9

EVARISTO, R.; FENEMA, P. C. A typology of project management: emergence and evolution of new forms. International Journal of Project Management, v. 17, n. 5, p. 275-281, 1999. http://dx.doi.org/10.1016/S02637863(98)00041-6

FORTUNE, J.; WHITE, D. Framing of project critical success factors by a system model. International Journal of Project Management, v. 24, n. 1, p. 53-65, 2006. http:// dx.doi.org/10.1016/j.jproman.2005.07.004

HÖLZING,J.DieKano-TheoriederKundenzufriedenheitsmessung: Eine theoretische und empirische Überprüfung. Wiesbaden: GWV Fachverlage GmbH, 2008

KANO, N. et al. Attractive quality and must be quality. Quality, v. 14, p. 39-48, 1984.

KERZNER, H. Gestão de Projetos. 2. ed. Porto Alegre: Bookman, 2006.

LOPES, D. Critérios de avaliação do desempenho de gerenciamento de projetos: uma abordagem de estudos de casos. Dissertação - Engenharia de Produção da Escola Politécnica da USP. São Paulo, p. 111, 2009

McFARLAN, F. W. Information technology changes the way you compete. Harvard Business Review, n. 84308, 1984.

MUNNS, A. K.; BJEIRMI, B. F. The role of project management in achieving project success. International Journal of Project Management, v.14, n. 2, p. 81-87, 1996. http:// dx.doi.org/10.1016/0263-7863(95)00057-7

MURPHY, D.; BAKER, N.; Fisher, D. Determinants of Project Success. Boston: Boston College, National Aeronautics and Space Administration, 1974.

PATAH, L. A.; CARVALHO, M. M. Sucesso em projetos a partir de uma perspectiva contingencial. In: RABECHINI JUNIOR, R.; CARVALHO, M. M. (Orgs.). Gerenciamento de Projetos na Prática 2: Casos Brasileiros. São Paulo: Ed. Atlas, 2009.
PINTO, J. K.; SLEVIN, D. P. Critical factors in successful project implementation. IEEE Transaction on Engineering Management, v. 34, n. 1, p. 22-28, 1987.

PINTO, J. K.; SLEVIN, D. P. Project success: definitions and measurement techniques. Project Management Journal, v. 19, n. 1, p. 67-72, 1988a.

PINTO, J. K.; SLEVIN, D. P. Critical success factors across the project life cycle. Project Management Journal, v. 19, n. 3, p. 67-75, 1988b.

PINTO, J. K.; SLEVIN, D. P. Critical success factors in R\&D projects. Research Technology Management, p. 31-35, 1989.

RABECHINI JUNIOR, R.; CARVALHO, M. M. Gestão de projetos inovadores em uma perspectiva contingencial: Análise teórico-conceitual e proposição de um modelo. Revista de Administração e Inovação, v. 6, n. 3, p. 63-78, set/dez. 2009.

ROCKART, J. F. Chief executives define their own data needs. Harvard Business Review, p. 81-93, 1979.

SABBAG, P. Y. The Nature of Projects: a tool for improving management. In: ANNUAL PROJECT INSTITUTE SEMINARS AND SYMPOSIUM, 20., 1999, Pennsylvania. Proceedings... Pennsylvania, 1999.

SHENHAR, A. J. One size does not fit all projects: Exploring classical contingency domains. Management Science, v. 47, n. 3, p. 394-414, 2001. http://dx.doi.org/10.1287/ mnsc.47.3.394.9772

SHENHAR, A. J. et al. Refining the search for project success factors: a multivariate, typological approach. $R \& D$ Management, v. 32, n. 2, p. 111-126, 2002. http://dx.doi. org/10.1111/1467-9310.00244

SHENHAR, A. J.; DVIR, D. Reinventing project management: The diamond approach to successful growth and innovation. Boston: Harvard Business School Press, 2007.

SLEVIN, D. P.; PINTO, J. K. The project implementation profile: New tool for project managers. Project Management Journal, v. 17, n. 4, p. 57-70, 1986.

VERZUH, E. MBA Compacto: Gestão de Projetos. Tradução de André de L. Cardoso. 4. ed. Rio de Janeiro: Campus, 2000.

\title{
Analysis of critical success factors for projects: a case study in the retail industry
}

\begin{abstract}
Based on the literature of critical success factors and project success, this study aimed to establish a relationship between both from a perspective that concerns different types of projects. In the context of a retail company, the empirical research was lead in two phases: a project management diagnosis through document analysis and interviews; and a survey with closed questions applied to the staff involved in the activity of projects in the company. Forty-three usable responses were obtained. One of the results indicates the significant impact of project typology on the performance of most critical success factors, highlighting the importance of defining objective criteria to classify projects. Moreover, it was possible to visualize, graphically, the relationship between each success dimension and its benefits to the firm through the modified Kano-diagram of customer satisfaction, previously used in this paper for the literature synthesis.
\end{abstract}

Keywords

Project success. Critical success factors for projects. Project classification. Kano diagram. 